\title{
Randomized crossover trial of silicone hydrogel contact lenses
}

\author{
María García-Montero $^{\mathrm{a}, *}$, Laura Rico-del-Viejo ${ }^{\mathrm{a}}$, Clara Llorens-Quintana ${ }^{\mathrm{b}}$, \\ Amalia Lorente-Velázquez ${ }^{a}$, Jose Luis Hernández-Verdejo ${ }^{a}$, David Madrid-Costa ${ }^{a}$ \\ ${ }^{\text {a }}$ Department of Optometry and Vision, Faculty of Optics and Optometry, Complutense University of Madrid, Madrid, Spain \\ ${ }^{\mathrm{b}}$ Department of Biomedical Engineering, Faculty of Fundamental Problems of Technology, Wroclaw University of Science and Technology, Wroclaw, Poland
}

\section{A R T I C L E I N F O}

\section{Keywords:}

Ocular surface

Tear film

Contact lens

Silicone hydrogel

Contact lens discomfort

\begin{abstract}
A B S T R A C T
Purpose: The aim of the current study is to assess, using new technologies, the interaction of four monthly silicone hydrogel contact lenses on the ocular surface and the comfort over 15 days of use.

Methods: Prospective cross-over, randomized and double-masked study including four materials (lotrafilcon-B, samfilcon-A , comfilcon-A and filcom-V3). Clinical examination was performed in the following order: tear meniscus height, first break-up of the tear film, the average time of all tear film breakup incidents, bulbar redness, limbal redness (Keratograph 5M ,Oculus, Germany); central corneal thickness (Pentacam, Oculus, Germany), thermography values (FLIR A325; FLIR Systems Inc., USA), and slit-lamp evaluations, including ocular surface staining. Finally, subjective comfort was obtained from Contact Lens Dry Eye Questionnaire-8. Results: The impact of contact lens wear on the ocular surface didn't show statistically significant changes over time except for corneal and conjunctival staining grades on day 15 compared to day 1 for the comfilcon A group ( $P=.003$ and $P=.01$, respectively). Contact lens stability and impact on the ocular surface during contact lens wear didn't show statistically significant changes over time except in the case of the comfilcon A material with respect to the irritation item $(P=.01)$.

Conclusions: These results suggest that the impact of monthly silicone hydrogel contact lens materials on the ocular surface after and during contact lens wear, contact lens stability over time, and subjective comfort did not reveal any significant changes over 15 days of use for any of the materials.
\end{abstract}

\section{Introduction}

The interactions of contact lenses with the tear film and ocular surface, as well as with environmental factors, play a critical role, both in successful contact lens wear and the development of contact lens discomfort $[1,2]$. With contact lens wear, the tear film undergoes extensive biophysical and biochemical changes, which have the potential to influence tear function and/or contact lens tolerance [3]. At the same time, it should be kept in mind that today's lifestyle exposes millions of individuals worldwide to artificially-controlled, low humidity, highvelocity airflow environments in office buildings and automobiles, with extended use of visual display units, such as tablets, computers and mobile phones. Such exposure may increase the tear film evaporation rate with the concomitant intensification of contact lens-related discomfort [4].

Contact lens discomfort is one of the major causes of contact lens wear dropout $[1,2]$. The key factors affecting contact lens comfort are the interaction of the contact lens (material and design) with patients' ocular surface, and the external environment. Laboratories are therefore working on new materials, designs and surface treatments to find the best tolerance for contact lens wearers $[5,6]$. Lens type differences in tear film surface quality have been found in in-vivo measurements $[7,8]$.

There is a need for new studies that assess the interaction between new contact lens materials on the ocular surface and their comfort. These can be carried out with current technology, that allows the objective and reliable assessment of changes in the ocular surface, such as ocular redness [9] and tear film stability [10]. Furthermore, recently it has been published that there is an association between ocular surface temperature and tear film stability in wearers of soft contact lenses [11], suggesting that ocular surface temperature measurements can be used to evaluate tear film stability in soft contact lens wearers.

The aim of this crossover prospective study was to assess the interaction of four soft contact lens materials on the ocular surface by means of classical clinical tests, using non-invasive ocular imaging and ocular surface temperature measurements to study the changes, both in

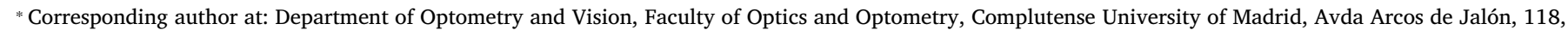
28037, Madrid, Spain.

E-mail address: mgarc01@ucm.es (M. García-Montero). 
the ocular surface and the contact lens, over 15 days of use. Subjective comfort outcomes were also assessed.

\section{Methods}

A prospective crossover randomized and double-masked study was carried out at the Faculty of Optics and Optometry of the Complutense University of Madrid. It was reviewed and approved by the Institutional Review Board of the Optometry Clinic, and all the procedures followed the tenets of the Declaration of Helsinki. Informed consent was obtained from all patients after the purpose and the possible consequences of the study had been explained to them. Inclusion criteria were an age range from 18 to 40 years, currently contact lens wearers, with a cylinder refractive error of $<0.50 \mathrm{D}$ and a spherical refractive error ranging from -4.00 to $+4.00 \mathrm{D}$. Exclusion criteria included an active ocular allergy, refractive surgery or systemic medication known to affect tear film production.

\subsection{Contact lenses}

The study was carried out using four monthly silicone hydrogel contact lenses. The materials were lotrafilcon B, samfilcon A, comfilcon $\mathrm{A}$ and filcom V3. The lens parameters are shown in Table 1.

\subsection{Measurements}

\subsubsection{Keratograph $5 M$ automated}

All the participants underwent imaging with the Keratograph $5 \mathrm{M}$ (K5M; Oculus GmbH, Wetzlar, Germany) equipped with the modified tear film scanning function. Three measurements of the tear meniscus height (TMH), the first break-up of the tear film (NIKBUT-first), the average time of all tear film break-up incidents (NIKBUT-avg), the bulbar redness (BR) and the limbal redness (LR) were obtained automatically from Oculus K5 M software according to the manufacturer's instructions. This system generates a redness score automatically, which is based on the area percentage ratio between the vessels and the rest of the analyzed area. For instance, if the ratio is $16 \%$, then the score is 1.6 [9]

\subsubsection{Pentacam}

Three measurements of central corneal thickness (CCT) were obtained by a rotating Scheimpflug camera system for anterior segment analysis (Pentacam, Oculus GmbH, Wetzlar, Germany).

\subsubsection{Thermography}

In order to determine the ocular surface temperature, thermography recordings from the eye and its surroundings were conducted, using a non-contact infrared thermography camera (FLIR A325; FLIR Systems Inc., USA). This thermal camera has an image resolution of $320 \times 240$ pixels, a thermal sensitivity of $50 \mathrm{mK}$ with an accuracy of $\pm 2 \%$ and a temperature range from $-20^{\circ} \mathrm{C}$ to $120^{\circ} \mathrm{C}$. For tear film temperature recordings the emissivity was set to 0.975 . [12] The camera was properly mounted on a chin rest with an approximation lens that allowed measurements to be taken at a distance of $5 \mathrm{~cm}$ from the subject's eye. Data acquisition was done with a temporal frequency of $20 \mathrm{~Hz}$. In order to perform the measurements the patient was instructed to rest his or her head on the chin rest, look straight forward and blink normally for a period of $40 \mathrm{~s}$.

Temperature values were obtained using software provided by the FLIR manufacturer and exported to be analyzed offline using Matlab R2017b (Version 9.3). Those frames that were affected by blinks were removed for the analyses. The area where the analyses were performed was manually delineated and kept constant for each sequence.

In order to characterize dynamic temperature changes in each sequence different parameters were computed. The variables obtained from the analyses were ocular surface mean temperature (OSMT), initial ocular surface temperature at zero seconds of register (Start-OST) and the final ocular surface temperature (End-OST) during the last seconds of register.

\subsubsection{Slit lamp}

Slit lamp evaluations included corneal and conjunctival staining. Slit lamp examination of the cornea and conjunctiva was performed under diffuse illumination using $\times 10-\times 16$ magnification. Staining scores were recorded according to the Oxford scheme (range 0-5). [13] Two minutes after instilling a sodium fluorescein dye (Bausch and Lomb, Rochester, NY, USA), corneal staining was graded using a cobalt blue filter over the slit-lamp and Kodak Wratten 12 yellow barrier filter. Conjunctival staining was assessed using lissamine green with Kodak Wratten 92. All the participants underwent slit lamp examination to observe the ocular surface staining.

\subsubsection{Symptom questionnaire}

The Contact Lens Dry Eye Questionnaire-8 (CLDEQ-8) was applied in order to quantify discomfort in contact lens wearers. Scoring was calculated for each item according the authors' instructions. [14] The items evaluated were comfort, dryness, blurry vision, irritation, grittiness, foreign body sensation, burning, photophobia and itching for each contact lens. Changes to the CLDEQ-8 score are considered to reflect a lens wearer's global opinion of lens comfort [15].

Table 2 shows a summary of parameter abbreviations used in the text.

\subsection{Study protocol}

The study protocol is shown in Fig. 1. The study was conducted over five consecutive weeks. Previously one week of washout without any contact lens was required to participants. During the first two weeks, each subject used one contact lens in the right eye and another different contact lens in the left eye. After a week of wash-out, another two

Table 1

Contact lens parameters for silicone hydrogel materials used in the study.

\begin{tabular}{|c|c|c|c|c|}
\hline Material & Lotrafilcon B & Samfilcon A & Comfilcon A & Filcom V3 \\
\hline Laboratory & Alcon & Bausch\&Lomb & CooperVision & Mark'Ennovy \\
\hline Commercial name & Air Optix HydraGlyde & Ultra & Biofinity & Blu:gen \\
\hline Base curve (mm) & 8.60 & 8.50 & 8.60 & $\begin{array}{l}6.50-9.80 \\
\text { (step } 0.30 \text { ) }\end{array}$ \\
\hline Diameter (mm) & 14.20 & 14.20 & 14.00 & $\begin{array}{l}11.50-16.50 \\
\text { (step } 0.50 \text { ) }\end{array}$ \\
\hline $\begin{array}{l}\text { Oxygen Transmissibility } \\
\text { (Dk/t) }\end{array}$ & 138 & 163 & 160 & 50 \\
\hline $\begin{array}{l}\text { Water content } \\
\qquad(\%)\end{array}$ & 33 & 46 & 48 & 75 \\
\hline $\begin{array}{l}\text { Modulus } \\
\quad(\mathrm{MPa})\end{array}$ & 1.0 & 0.70 & 0.75 & 0.25 \\
\hline
\end{tabular}


Table 2

Summary of the abbreviations used.

\begin{tabular}{ll}
\hline Parameter & Abbreviations \\
\hline Tear meniscus height & TMH \\
First break-up of the tear film & NIKBUT-first \\
Average time of all tear film break-up incidents & NIKBUT-avg \\
Bulbar redness & BR \\
Limbal redness & LR \\
Central corneal thickness & CCT \\
Ocular surface mean temperature & OSMT \\
Initial ocular surface temperature at zero seconds of register & Start-OST \\
Final ocular surface temperature & End-OST \\
Contact Lens Dry Eye Questionnaire-8 & CLDEQ-8 \\
\hline
\end{tabular}

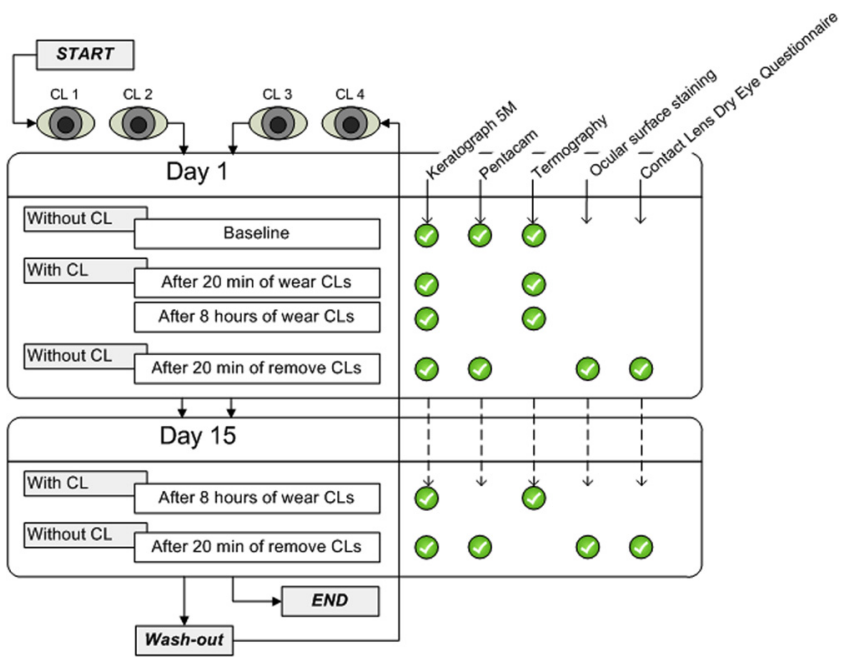

Fig. 1. Clinical protocol. Randomized assigned contact lenses. Repeated measures for each visit.

CL: contact lens; min: minutes.

contact lenses were assigned to the right and left eye for two more weeks. Contact lenses were assigned randomly. Measurements were taken for each pair of contact lenses on the first and last day of wear (15 days). On the first day of wear, measurements were taken before contact lens insertion (baseline), at $20 \mathrm{~min}$ and $8 \mathrm{~h}$ of wear, and after the lens was removed. On the last day of wear (after 15 days of use), measurements were taken at $8 \mathrm{~h}$ of wear and after the lens was removed. Measurements without contact lenses were registered $20 \mathrm{~min}$ after the lens was removed.

Clinical examination was performed in the following order to minimize the effect of the previous measurement: TMH, NIKBUT-first, NIKBUT-avg, BR and LR (Keratograph 5M, Oculus, Germany); CCT (Pentacam, Oculus, Germany), thermography values (FLIR A325; FLIR Systems Inc., USA), and slit-lamp evaluations, including ocular surface staining. Finally, information on the level of satisfaction with the contact lens was obtained from a CLDEQ-8 for each eye. A 5-minute interval between each test was established, and all tests were performed in the same order. All the measurements were performed by the same examiner in a room with controlled temperature $\left(24 \pm 1^{\circ} \mathrm{C}\right)$ and humidity $(38 \% \pm 2 \%)$.

The Keratograph $5 \mathrm{M}$ parameters were taken at every visit established in the study protocol. CCT was measured on the first day under baseline conditions, and following lens removal after $8 \mathrm{~h}$ of use on the first and the last day (15 days). Thermography values were obtained on the first day under baseline conditions, after $20 \mathrm{~min}$ and $8 \mathrm{~h}$ of wear, and on the last day after $8 \mathrm{~h}$ of wear (after 15 days of use). Ocular surface staining and the CLDEQ- 8 were registered after $8 \mathrm{~h}$ of wear on the first day and the last day (15 days).

All subjects used the same solutions to care for the lenses (Optifree
Express Mds, Alcon Laboratories, Inc., Fort Worth, TX, USA).

The results were grouped into two analyses. One assessed the impact of the contact lens on the ocular surface by comparing measurements taken without contact lenses (at baseline, day 1 without contact lenses after $8 \mathrm{~h}$ of use, and day 15 without contact lenses after $8 \mathrm{~h}$ of use). The other assessed contact lens stability and the impact on the ocular surface by comparing measurements taken during the wearing of contact lenses (day 1 at $20 \mathrm{~min}$ and $8 \mathrm{~h}$ of contact lens wear, and day 15 at $8 \mathrm{~h}$ of contact lens wear).

\subsection{Data analysis}

Statistical analyses were performed using SPSS statistic software, version 22.0 (SPSS Inc., Chicago, IL, USA). First, a descriptive analysis was carried out to establish the mean results and standard deviations for each material and visit. The Shapiro-Wilks test for normality was applied for each variable analyzed. To analyze the comparisons of the same subjects under different conditions, repeated measures (withinsubjects) ANOVA test and Friedman test was applied with the Greenhouse-Geisser correction for parametric and non-parametric distributions respectively. The significance level used was $P<.05$.

\section{Results}

Fifteen subjects (12 men and 3 women; mean age $24.1 \pm 2.2$ years; age range 21-29 years) were enrolled and all the protocols were completed.

\subsection{Impact of contact lens wear on the ocular surface}

Table 3 shows the results of the ocular surface parameters measured

Table 3

Descriptive statistics and comparative analysis for clinical parameters measured with Keratograph $5 \mathrm{M}$ without contact lenses: the first day under baseline conditions and after the lenses were removed after $8 \mathrm{~h}$ of use on the first and last day (15 days). Data are expressed as the (mean \pm standard deviation). Impact of the contact lens on the ocular surface.

\begin{tabular}{lllll}
\hline & Baseline & Day $1-8 \mathrm{~h}$ & Day $15-8 \mathrm{~h}$ & \\
\hline & TMH & & & $P$ \\
Lotrafilcon B & $0.27 \pm 0.12$ & $0.30 \pm 0.15$ & $0.25 \pm 0.08$ & .17 \\
Samfilcon A & $0.30 \pm 0.08$ & $0.32 \pm 0.13$ & $0.29 \pm 0.08$ & .13 \\
Comfilcon A & $0.28 \pm 0.11$ & $0.32 \pm 0.19$ & $0.24 \pm 0.06$ & .11 \\
Filcom V3 & $0.30 \pm 0.12$ & $0.34 \pm 0.10$ & $0.29 \pm 0.06$ & .08 \\
& NIKBUT-first & & & $P$ \\
Lotrafilcon B & $10.64 \pm 5.80$ & $9.41 \pm 5.74$ & $10.19 \pm 5.33$ & .71 \\
Samfilcon A & $12.09 \pm 6.93$ & $10.83 \pm 5.37$ & $8.84 \pm 4.00$ & .22 \\
Comfilcon A & $11.78 \pm 6.52$ & $9.00 \pm 4.71$ & $9.28 \pm 5.64$ & .12 \\
Filcom V3 & $9.39 \pm 5.17$ & $11.65 \pm 4.16$ & $11.71 \pm 7.17$ & .28 \\
& NIKBUT-avg & & & $P$ \\
Lotrafilcon B & $13.19 \pm 5.96$ & $12.19 \pm 6.09$ & $12.82 \pm 5.33$ & .30 \\
Samfilcon A & $14.43 \pm 6.91$ & $14.03 \pm 5.37$ & $12.41 \pm 4.48$ & .46 \\
Comfilcon A & $13.59 \pm 5.91$ & $11.84 \pm 6.13$ & $12.01 \pm 5.56$ & .08 \\
Filcom V3 & $12.72 \pm 4.60$ & $14.50 \pm 4.83$ & $14.72 \pm 6.73$ & .55 \\
& BR & & & $P$ \\
Lotrafilcon B & $1.91 \pm 0.52$ & $2.21 \pm 0.66$ & $1.96 \pm 0.67$ & .14 \\
Samfilcon A & $1.74 \pm 0.63$ & $2.16 \pm 0.78$ & $1.68 \pm 0.70$ & .21 \\
Comfilcon A & $1.89 \pm 0.46$ & $2.02 \pm 0.58$ & $1.93 \pm 0.53$ & .65 \\
Filcom V3 & $1.66 \pm 0.51$ & $1.78 \pm 0.58$ & $1.83 \pm 0.49$ & .70 \\
& LR & & & $P$ \\
Lotrafilcon B & $1.16 \pm 0.49$ & $1.54 \pm 0.77$ & $1.26 \pm 0.53$ & .13 \\
Samfilcon A & $1.15 \pm 0.82$ & $1.70 \pm 0.98$ & $1.27 \pm 0.86$ & .23 \\
Comfilcon A & $1.21 \pm 0.49$ & $1.43 \pm 0.62$ & $1.18 \pm 0.57$ & .26 \\
Filcom V3 & $1.13 \pm 0.71$ & $1.16 \pm 0.58$ & $1.28 \pm 0.56$ & .75 \\
\hline
\end{tabular}

Units: Tear meniscus height (mm); break-up tear film (seconds); redness (ratio between the vessels and the rest of the analyzed area).

TMH: tear meniscus height; NIKBUT-avg: average time of all tear film break-up; NIKBUT-first: first break-up of the tear film; BR: bulbar redness; LR: limbal redness. 


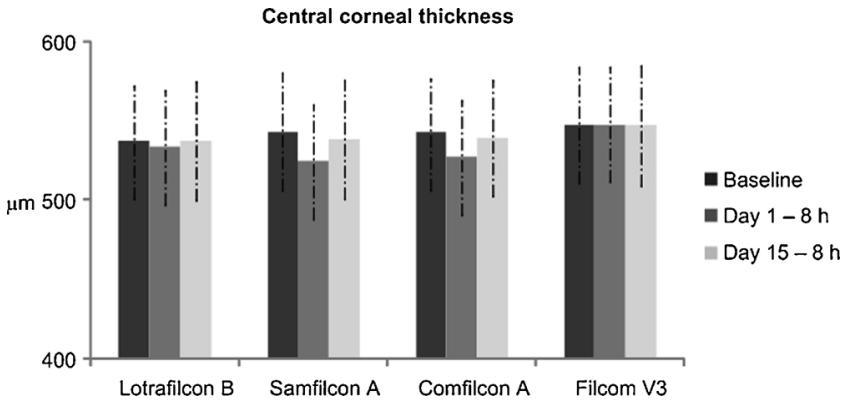

Fig. 2. Comparison of ocular staining (Oxford Scale; grade 0-5) after contact lens wear between the first and 15th day of use. (A) Increased corneal staining at 15 days of use with respect to one day of use for comfilcon A. (B) Conjunctival staining increased at 15 days of use with respect to one day of use for comfilcon. (*) $P<.05$.

with Keratograph $5 \mathrm{M}$ (TMH, NIKBUT-first, NIKBUT-avg, BR and LR) at baseline and without the contact lens after 1 day and 15 days of use. There were no statistically significant differences between the three visits in any ocular surface parameters for any contact lens.

Fig. 2 shows the changes in corneal (Fig. 2A) and conjunctival staining (Fig. 2B) over wear time for each contact lens. Corneal and conjunctival staining grades were only significantly higher on day 15 compared to day 1 for the comfilcon A group $(P=.003$ and $P=.01$, respectively).

Fig. 3 shows the CCT values before and after contact lens wear. There were no statistically significant changes over time.

\subsection{Contact lens stability and impact on the ocular surface during contact lens wear}

Table 4 shows the TMH, NIKBUT-first, NIKBUT-avg, BR and LR during contact lens wear. There were no statistically significant differences in any material over the contact lens wear time.

Descriptive statistics and the comparative analysis for the clinical parameters measured, obtained with thermography recordings, are summarized in Table 5. After contact lens wear, no changes were found in the OSMT, Start-OST and End-OST for any of the materials.

The results of the CLDEQ- 8 are summarized in Table 6. This shows the descriptive values for each questionnaire item. There were no statistically significant differences in any item except in the case of the comfilcon A material with respect to the irritation item $(P=.01)$. Comfilcon A showed a higher score for irritation after $8 \mathrm{~h}$ of use on day 15 than on day $1[(0.17 \pm 0.19)$ vs $(0.06 \pm 0.12)$ respectively].

\section{Discussion}

Wearing contact lenses implies an interaction between the lens and
A

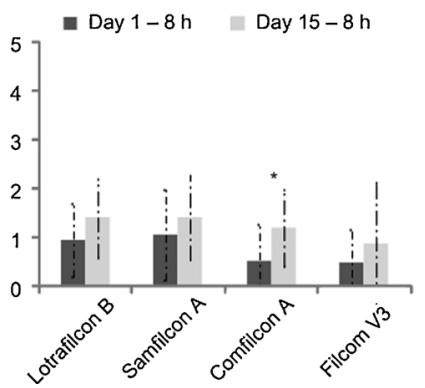

B Conjuntival staining

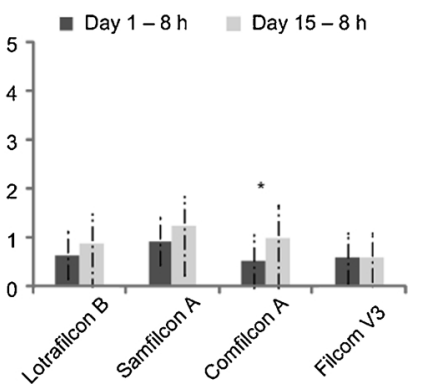

Fig. 3. Impact of contact lens wear on central corneal thickness. Comparison of central corneal thickness values before and after contact lens wear; baseline conditions and after contact lens wear on the first and 15th day of use.
Table 4

Descriptive statistics and comparative analysis for clinical parameters measured with Keratograph $5 \mathrm{M}$ during contact lens wear: at $20 \mathrm{~min}$ and $8 \mathrm{~h}$ of use on the first day, and at $8 \mathrm{~h}$ of use on the last day (15 days). Data are expressed as the (mean \pm standard deviation). Contact lens stability and impact on the ocular surface during contact lens wear.

\begin{tabular}{lllll}
\hline & Day $1-20 \mathrm{~min}$ & Day $1-8 \mathrm{~h}$ & Day $15-8 \mathrm{~h}$ & \\
\hline & TMH & & & $P$ \\
Lotrafilcon B & $0.27 \pm 0.20$ & $0.25 \pm 0.08$ & $0.23 \pm 0.07$ & .43 \\
Samfilcon A & $0.27 \pm 0.08$ & $0.29 \pm 0.08$ & $0.28 \pm 0.08$ & .55 \\
Comfilcon A & $0.25 \pm 0.10$ & $0.24 \pm 0.08$ & $0.24 \pm 0.06$ & .77 \\
Filcom V3 & $0.31 \pm 0.11$ & $0.28 \pm 0.14$ & $0.27 \pm 0.08$ & .42 \\
& NIKBUT-first & & & $P$ \\
Lotrafilcon B & $9.72 \pm 5.67$ & $9.64 \pm 3.77$ & $9.36 \pm 4.28$ & .91 \\
Samfilcon A & $8.86 \pm 5.87$ & $8.94 \pm 4.84$ & $8.63 \pm 5.00$ & .93 \\
Comfilcon A & $11.11 \pm 6.60$ & $10.93 \pm 5.36$ & $12.58 \pm 5.49$ & .42 \\
Filcom V3 & $12.78 \pm 5.41$ & $13.19 \pm 5.63$ & $11.45 \pm 7.09$ & .74 \\
& NIKBUT-avg & & & $P$ \\
Lotrafilcon B & $16.21 \pm 3.55$ & $14.78 \pm 3.99$ & $15.61 \pm 3.32$ & .22 \\
Samfilcon A & $15.09 \pm 4.69$ & $15.45 \pm 3.69$ & $15.47 \pm 3.55$ & .91 \\
Comfilcon A & $16.48 \pm 4.91$ & $15.55 \pm 3.63$ & $17.89 \pm 3.75$ & .17 \\
Filcom V3 & $18.49 \pm 3.20$ & $16.48 \pm 5.04$ & $15.92 \pm 4.86$ & .37 \\
& BR & & & $P$ \\
Lotrafilcon B & $1.83 \pm 0.57$ & $2.02 \pm 0.60$ & $1.76 \pm 0.55$ & .50 \\
Samfilcon A & $1.71 \pm 0.54$ & $1.91 \pm 0.68$ & $1.49 \pm 0.36$ & .33 \\
Comfilcon A & $1.62 \pm 0.58$ & $1.69 \pm 0.61$ & $1.72 \pm 0.67$ & .76 \\
Filcom V3 & $1.49 \pm 0.37$ & $1.61 \pm 0.29$ & $1.54 \pm 0.29$ & .66 \\
& LR & & & .36 \\
Lotrafilcon B & $0.99 \pm 0.61$ & $1.16 \pm 0.69$ & $0.95 \pm 0.63$ & .36 \\
Samfilcon A & $0.97 \pm 0.75$ & $1.09 \pm 0.79$ & $0.80 \pm 0.44$ & .19 \\
Comfilcon A & $1.00 \pm 0.48$ & $1.02 \pm 0.56$ & $0.87 \pm 0.57$ & .61 \\
Filcom V3 & $1.26 \pm 0.71$ & $1.11 \pm 0.42$ & $1.29 \pm 0.55$ & .81 \\
\hline & & & & \\
\hline
\end{tabular}

Units: Tear meniscus height (mm); break-up tear film (seconds); redness (ratio between the vessels and the rest of the analyzed area).

TMH: tear meniscus height; NIKBUT-avg: average time of all tear film break-up; NIKBUT-first: first break-up of the tear film; BR: bulbar redness; LR: limbal redness.

the ocular surface. This interaction can produce tear film and ocular surface alterations and discomfort $[16,17]$ which can be affected by several factors associated with material characteristics, such as lubricity and water content, and wear time. Silicone hydrogel lenses for daily wear show significant improvements in clinical signs and subjective symptoms when compared to conventional hydrogel lens for daily wear $[18,19]$. Therefore, in view of the need to know how silicone hydrogel materials affect the ocular surface and subjective comfort, the aim of this current study was to evaluate the impact of four different monthly contact lenses on lens stability, the ocular surface and contact lens comfort over 15 days of use using new technologies to determine if they were superior to traditional slit lamp findings.

Tear film volume can be quantitatively assessed by measuring the tear meniscus height [3]. During contact lens wear, tear meniscus height can be affected by different factors, and some authors have concluded that tear volume gradually decreases with lens wear. Chen et al. (2011) monitored tear meniscus volumes (using anterior segment optical coherence tomography imaging) for long-term daily contact lenses (etafilcon A) at 2, 4, 6, 8 and $10 \mathrm{~h}$, and observed a decrease in tear meniscus volume over $10 \mathrm{~h}$ of contact lens wear [20]. Wolffsohn et al. measured tear meniscus height by modified topographer for three daily disposable silicone hydrogel contact lenses over one day at 8,12 , and $16 \mathrm{~h}$ [21]. The findings of this study with daily contact lenses showed that the interaction of the lenses on the tear meniscus height differed between lens types, however there was no decrease in the tear reservoir from 8 to $16 \mathrm{~h}$ of wear overall. The results of the current research show that the studied contact lenses did not affect tear meniscus height during the 15 days of contact lens wear. Another interesting factor to consider is whether the use of contact lenses affects tear production once the contact lens is removed. Nagahara et al reported decreases in the tear meniscus height $20 \mathrm{~min}$ after contact lens 
Table 5

Descriptive statistics and comparative analysis for clinical parameters measured, obtained with thermographic recordings under baseline conditions and with contact lens wear at $20 \mathrm{~min}$ and $8 \mathrm{~h}$ of use on the first day, and after $8 \mathrm{~h}$ of use on the last day (15 days). Data are expressed as the (mean \pm standard deviation). Contact lens stability and impact on the ocular surface during contact lens wear.

\begin{tabular}{|c|c|c|c|c|c|}
\hline & Baseline & Day $1-20 \mathrm{~min}$ & Day $1-8 \mathrm{~h}$ & Day $15-8 h$ & \\
\hline & Lotrafilcon B & & & & $P$ \\
\hline OSMT & $35.13 \pm 0.99$ & $34.54 \pm 1.15$ & $34.89 \pm 1.17$ & $34.83 \pm 1.08$ & .13 \\
\hline Start-OST & $35.27 \pm 0.94$ & $34.74 \pm 1.09$ & $35.02 \pm 1.07$ & $35.01 \pm 0.99$ & .14 \\
\hline \multirow[t]{2}{*}{ End-OST } & $35.08 \pm 1.02$ & $34.50 \pm 1.19$ & $34.80 \pm 1.20$ & $34.80 \pm 1.08$ & .15 \\
\hline & Samfilcon A & & & & $P$ \\
\hline OSMT & $35.31 \pm 0.65$ & $34.63 \pm 0.85$ & $35.03 \pm 0.87$ & $34.91 \pm 092$ & .17 \\
\hline Start-OST & $35.31 \pm 0.65$ & $34.73 \pm 0.76$ & $35.13 \pm 0.75$ & $35.01 \pm 0.82$ & .22 \\
\hline \multirow[t]{2}{*}{ End-OST } & $35.31 \pm 0.68$ & $34.57 \pm 0.89$ & $34.98 \pm 0.92$ & $34.86 \pm 0.95$ & .16 \\
\hline & Comfilcon A & & & & $P$ \\
\hline OSMT & $34.82 \pm 1.12$ & $34.20 \pm 1.12$ & $34.84 \pm 1.24$ & $34.47 \pm 1.35$ & .14 \\
\hline Start-OST & $34.91 \pm 1.03$ & $34.39 \pm 1.05$ & $34.92 \pm 1.16$ & $34.63 \pm 1.21$ & .15 \\
\hline \multirow[t]{2}{*}{ End-OST } & $34.76 \pm 1.13$ & $34.13 \pm 1.16$ & $34.78 \pm 1.29$ & $34.41 \pm 1.37$ & .10 \\
\hline & Filcom V3 & & & & $P$ \\
\hline OSMT & $35.16 \pm 0.91$ & $34.63 \pm 1.27$ & $35.05 \pm 1.27$ & $35.01 \pm 1.23$ & .19 \\
\hline Start-OST & $35.24 \pm 0.83$ & $34.81 \pm 1.12$ & $35.23 \pm 1.15$ & $35.14 \pm 1.08$ & .23 \\
\hline End-OST & $35.12 \pm 0.94$ & $34.57 \pm 1.32$ & $34.99 \pm 1.31$ & $34.93 \pm 1.30$ & .20 \\
\hline
\end{tabular}

Units: ocular surface $\left({ }^{\circ} \mathrm{C}\right)$.

OSMT: ocular surface mean temperature; Start-OST: initial ocular surface temperature at zero seconds of register; End-OST: final ocular surface temperature in the last seconds of register.

(nelfilcon A and lotrafilcon A) insertion using anterior segment optical coherence tomography imaging [22]. On the other hand, others studies did not find significant differences in tear meniscus height after contact lens insertion [23,24]. These results are accordance with the results of the current study, which did not find differences in tear meniscus height when this was compared with the baseline situation, before contact lens wear, and on the first and 15th day of use.

Another important aspect to consider during contact lens wear is tear film stability. The current study not only asses this parameter from a traditional perspective, through non-invasive tear break-up time [10], but also from a recently suggested new approach in which ocular surface temperature is obtained using infrared thermography [11].

It has been suggested that tear break-up time can be used as a tool to assess pre-corneal and pre-lens tear film quality for prescribing contact lenses. [25] Wolffsohn et al. showed that tear break-up time over the daily contact lens surface differed between lens types and may have a role in protecting the ocular surface [21]. Several authors did not observe any changes over time in contact lens wearers [21,26,27] using silicone hydrogel daily contact lenses at 8,12 , and $16 \mathrm{~h}$ of wear on one day. The findings of this study are in agreement with those found in earlier studies. However, it is important to note that in the present study the silicone hydrogel monthly contact lenses were evaluated over 15 days of use and no differences were found for any material. These findings suggest that the contact lenses studied do not have a significant impact on non-invasive tear break-up time during 15 days of wear.
It is generally accepted that the physical presence of a contact lens disrupts the normal tear film structure, in particular the lipid layer, and that this facilitates a more rapid loss of tear fluid by evaporation. [2] It has therefore been proposed that the measurement of ocular surface temperature before and during contact lens wear can be used as an index of tear film stability [11]. It seems that the greatest ocular temperature changes observed occur when measurements are taken immediately after contact lens insertion, and that the effect is greater with silicone hydrogel lenses than with conventional hydrogel [28]. However, there are many occasions when ocular responses to contact lens wear may initially be minimal, particularly with the advent of silicone hydrogel contact lenses [29].

Several authors have measured ocular surface temperature over contact lenses and reported a decrease in ocular surface temperature with contact lens wear $[16,21,28,30]$. A decrease of the order of $0.5^{\circ} \mathrm{C}$ was also found in a study performed over a model eye with 3 materials (lotrafilcon A, balafilcon A and etafilcon A) [31]. Itokawa et al. found lower differences that were not significant [11]. They published results with a silicone hydrogel material (delefilcon $\mathrm{A}$ ) that showed differences of $0.15 \pm 0.33^{\circ} \mathrm{C}$ between the baseline situation and $15 \mathrm{~min}$ of contact lens wear in a video register of $10 \mathrm{~s}$. The current results suggest that the pre-lens ocular surface temperature does not change over time. After $20 \mathrm{~min}$ of contact lens wear there was a non-significant decrease in ocular surface temperature, and values were similar preserved after $8 \mathrm{~h}$ on the first and the 15th days. These findings on non-invasive tear

Table 6

Descriptive statistics and comparative analysis for each questionnaire item obtained with the Contact Lens Dry Eye Questionnaire-8 after $8 \mathrm{~h}$ of use on the first day and last day (15 days). Data are expressed as the (mean \pm standard deviation).

\begin{tabular}{|c|c|c|c|c|c|c|c|c|c|c|c|c|}
\hline & \multicolumn{3}{|l|}{ Lotrafilcon B } & \multicolumn{3}{|l|}{ Samfilcon A } & \multicolumn{3}{|l|}{ Comfilcon A } & \multicolumn{3}{|l|}{ Filcom V3 } \\
\hline & $\begin{array}{l}\text { Day } 1 \\
8 \mathrm{~h}\end{array}$ & $\begin{array}{l}\text { Day } 15 \\
8 \mathrm{~h}\end{array}$ & $P$ & $\begin{array}{l}\text { Day } 1 \\
8 \mathrm{~h}\end{array}$ & $\begin{array}{l}\text { Day } 15 \\
8 \mathrm{~h}\end{array}$ & $P$ & $\begin{array}{l}\text { Day } 1 \\
8 \mathrm{~h}\end{array}$ & $\begin{array}{l}\text { Day } 15 \\
8 \mathrm{~h}\end{array}$ & $P$ & $\begin{array}{l}\text { Day } 1 \\
8 \mathrm{~h}\end{array}$ & $\begin{array}{l}\text { Day } 15 \\
8 \mathrm{~h}\end{array}$ & $P$ \\
\hline Comfort & $0.17 \pm 0.11$ & $0.16 \pm 0.09$ & .78 & $0.10 \pm 0.09$ & $0.14 \pm 0.10$ & .23 & $0.10 \pm 0.12$ & $0.12 \pm 0.10$ & .61 & $0.29 \pm 0.17$ & $0.22 \pm 0.16$ & .18 \\
\hline Dryness & $0.50 \pm 0.56$ & $0.68 \pm 0.46$ & .23 & $0.55 \pm 0.61$ & $0.93 \pm 0.78$ & .08 & $0.51 \pm 0.65$ & $0.66 \pm 0.86$ & .51 & $0.53 \pm 0.73$ & $0.68 \pm 0.55$ & .41 \\
\hline Blurry vision & $0.02 \pm 0.03$ & $0.03 \pm 0.03$ & .36 & $0.04 \pm 0.05$ & $0.03 \pm 0.04$ & .69 & $0.03 \pm 0.04$ & $0.04 \pm 0.04$ & .25 & $0.04 \pm 0.04$ & $0.04 \pm 0.04$ & .63 \\
\hline Irritation & $0.17 \pm 0.28$ & $0.19 \pm 0.19$ & .82 & $0.14 \pm 0.19$ & $0.20 \pm 0.35$ & .53 & $0.06 \pm 0.12$ & $0.17 \pm 0.19$ & $.01^{*}$ & $0.07 \pm 0.12$ & $0.07 \pm 0.09$ & .93 \\
\hline Grittiness & $0.03 \pm 0.07$ & $0.02 \pm 0.03$ & .41 & $0.02 \pm 0.04$ & $0.02 \pm 0.06$ & .83 & $0.01 \pm 0.03$ & $0.02 \pm 0.03$ & .13 & $0.08 \pm 0.11$ & $0.04 \pm 0.06$ & .22 \\
\hline Foreign body sensation & $0.36 \pm 0.43$ & $0.33 \pm 0.34$ & .59 & $0.09 \pm 0.12$ & $0.13 \pm 0.17$ & .41 & $0.09 \pm 0.21$ & $0.12 \pm 0.15$ & .76 & $0.57 \pm 0.45$ & $0.46 \pm 0.45$ & .43 \\
\hline Burning & $0.08 \pm 0.13$ & $0.08 \pm 0.12$ & .96 & $0.05 \pm 0.11$ & $0.01 \pm 0.04$ & .24 & $0.05 \pm 0.11$ & $0.07 \pm 0.12$ & .46 & $0.01 \pm 0.04$ & $0.01 \pm 0.04$ & .98 \\
\hline Photophobia & $0.21 \pm 0.33$ & $0.13 \pm 0.16$ & .36 & $0.10 \pm 0.15$ & $0.13 \pm 0.16$ & .66 & $0.23 \pm 0.34$ & $0.08 \pm 0.13$ & .11 & $0.15 \pm 0.19$ & $0.09 \pm 0.15$ & .38 \\
\hline Itching & $0.25 \pm 0.28$ & $0.25 \pm 0.16$ & $>.99$ & $0.21 \pm 0.23$ & $0.28 \pm 0.18$ & .20 & $0.17 \pm 0.25$ & $0.22 \pm 0.18$ & .60 & $0.13 \pm 0.19$ & $0.19 \pm 0.18$ & .27 \\
\hline
\end{tabular}

* Statistically significant differences. 
break-up time and ocular surface temperature suggest that the contact lens studied does not have a significant impact on tear film stability over 15 days of wear.

However, reported differences between studies on changes in ocular surface temperature could be attributed to the material types, modalities of wear and replacement times used in each one. In fact, Ooi et al. proposed that a contact lens with a higher water content has a lower steady state temperature than a lens with lower water content [31]. So, to study different water content wear is of interest and should be clarified in future studies.

In addition to tear film stability, it is crucial to study the health and integrity of the ocular surface. The present study assesses redness, ocular staining and corneal swelling.

In contact lens wearers, redness is related to the extent of oxygen transmissibility of contact lens materials and may indicate corneal hypoxia [32,33]. It is well known that hypoxia and mechanical actions induced by contact lens wear can cause corneal swelling. Ocular staining is also common in contact lens wearers and several factors have been identified as being related to this corneal staining [34].

In the current study, limbal and bulbar redness and central corneal thickness did not change after 15 days of contact lens wear for any of the lenses. Neither were there any changes in corneal and conjunctival staining, with the exception of Comfilcom A after 15 days of use. Both conjunctival staining (which typically presents with circumlimbal staining along the contour of the lens edge) and corneal staining were statistically significant. However, the values of $1.00 \pm 0.65$ and $1.20 \pm 0.77$ for conjunctival and corneal staining, respectively, after 15 days of contact lens wear were not considered clinically significant on a scale of $0-5^{\circ}$.

Some authors reported an increase in bulbar redness after $8 \mathrm{~h}$ of wear with somofilcon A and narafilcon A material [26]. Other studies showed no changes in redness. However, greater corneal and conjunctival staining was observed after $16 \mathrm{~h}$ of wear with filcon II-3 [21].

Cheung et al. concluded that levels of corneal staining and limbal and conjunctival injection were statistically insignificant when silicone hydrogel and hydrogel daily contact lenses were compared [35]. Vicente et al. reported that silicone hydrogel lenses have eliminated hypoxia [36].

The health and integrity of the ocular surface during and after contact lens wear was therefore maintained throughout the study for all the contact lenses examined.

Finally, the study of comfort allows to obtain a complete report of the effects of the considered contact lenses on subjective comfort.

Chalmers et al. concluded that users of silicone hydrogel contact lenses have a more positive use experience compared to users of hydrogel contact lenses [15], although other authors did not find any differences in comfort when both materials were compared [35].

In the current study, there seems to be no increase in the severity of symptoms in silicone hydrogel contact lens wearers after 15 days of use. These results are in accordance with the study by Dumbleton et al. [29] which examined overall comfort and any burning or dryness with scales of 0 to $100(0=$ worst rating, $100=$ best rating $)$ in galyfilcon $\mathrm{A}$, senofilcon A, lotrafilcon B, lotrafilcon A and balafilcon A materials. They concluded from their study that no changes could be found over a 2week wearing period.

On the other hand, other authors [20,21] have reported that ocular comfort ratings decreased with time during the day after $10 \mathrm{~h}$ of daily soft contact lens wear.

Contact lens wear time may play an important role in subjective symptoms of discomfort, however in this study no changes were observed when the degree of comfort was assessed after one day and fifteen days of contact lens wear. Hence, from a statistical point of view, we cannot know whether there really were no differences in the subjective outcomes or we do not have enough sample to find differences "The underpowered studies should be interpreted cautiously and the 'absence of evidence' in these studies should not be taken as 'evidence of absence"
[37]. But, considering the results at the 1 and 15 day visits for all lenses, it seems that there really were not differences.

Furthermore, the use of different questionnaires makes it difficult to summarize the results. The use of normalized questionnaires may be better in this case. The Contact Lens Dry Eye Questionnaire-8 [15] is therefore useful for identifying soft contact lens wearers who may benefit from lenses or lens care products designed to reduce symptoms of dryness with soft contact lens wear, and for tracking their progress in treatment trials. The results of this study essentially showed no effect over time considering the pre-lens baseline as control parameters. In order to study if new technology tests can quantifying the clinical signs in a superior way to traditional slit lamp observation, it could be appropriate including a low water content hydrogel lens. Another limitation of the current study is an insufficient size of the sample for symtomatology items, although the rest of variables have $>80 \%$ power using a paired $t$-test with a 0,050 two-sided significance level to detect a difference in means.

\section{Conclusions}

This double masked randomized study with 15 days of follow-up, in which non-invasive techniques such as Keratograph $5 \mathrm{M}$ and thermography were used to evaluate the ocular surface, was developed as an addition to classic corneal staining tests and comfort assessment with specific questionnaires, in order to understand the interaction of different silicone hydrogel materials on the ocular surface in more detail.

In conclusion, the impact of monthly silicone hydrogel contact lens materials (lotrafilcon B, samfilcon A, comfilcon A and filcom V3) on the ocular surface after and during contact lens wear, contact lens stability over time, and subjective comfort did not reveal any significant changes over 15 days of use for any of the materials, except for comfilcom A on ocular staining and the subjective irritation sensation on the 15th day of wear. However, these changes were not clinically significant.

The results of the current study suggest that these types of materials are able to maintain the integrity of different parameters of the ocular surface both during and after contact lens wear.

\section{Funding}

This research has received funding from the European Union's Horizon 2020 research and innovation programme under the Marie Skłodowska-Curie grant agreement No 642760

\section{Disclosure statements}

The authors have no commercial or proprietary interest in any concept or product described in this article.

\section{References}

[1] J.J. Nichols, M.D. Willcox, A.J. Bron, C. Belmonte, J.B. Ciolino, J.P. Craig, et al., The TFOS international workshop on contact lens discomfort: executive summary, Invest Ophthalmol Vis Sci 54 (2013) 7-13. TFOS.

[2] J.P. Craig, M.D. Willcox, P. Argüeso, C. Maissa, U. Stahl, A. Tomlinson, et al., The TFOS international workshop on contact lens discomfort: report of the contact lens interactions with the tear film subcommittee, Invest Ophthalmol Vis Sci 54 (2013) 123-156. TFOS.

[3] M.D. Willcox, P. Argueso, G.A. Georgiev, J.M. Holopainen, G.W. Laurie, T.J. Millar, et al., TFOS DEWS II tear film report, Ocul Surf 15 (2017) 366-403.

[4] A. López-de la Rosa, V. Martín-Montañez, A. López-Miguel, I. Fernández, M. Calonge, J.M. González-Méijome, et al., Ocular response to environmental variations in contact lens wearers, Ophthalmic Physiol Opt 37 (2017) 60-70.

[5] A. Tauste, E. Ronda, V. Baste, M. Bratveit, B.E. Moen, M.D. Segui Crespo, et al., Ocular surface and tear film status among contact Lens wearers and non-wearers who use vdt at work: comparing three different lens types, Int Arch Occup Environ Health 91 (2018) 327-335.

[6] J. Rex, T. Knowles, X. Zhao, J. Lemp, C. Maissa, S.S. Perry, et al., Elemental composition at silicone hydrogel contact lens surfaces, Eye Contact Lens 15 (January) (2018) [Epub ahead of print].

[7] D.H. Szczesna-Iskander, D.R. Iskander, S.A. Read, D. Alonso-Caneiro, Noninvasive 
in vivo assessment of Soft contact lens type on tear film surface quality, Invest Ophthalmol Vis Sci 53 (2012) 525-531.

[8] C. Llorens-Quintana, M. Mousavi, D. Szczessna-Iskander, D.R. Iskander, Non-invasive pre-lens tear film assessment with high-speed videokeratoscopy, Cont Lens Anterior Eye 41 (2018) 18-22.

[9] S. Wu, J. Hong, L. Tian, et al., Assessment of bulbar redness with a newly developed keratograph, Optom Vis Sci 92 (2015) 892-899.

[10] L. Tian, J.H. Qu, X.Y. Zhang, X.G. Sun, Repeatability and reproducibility of noninvasive keratograph $5 \mathrm{M}$ measurements in patients with dry eye disease, $\mathrm{J}$ Ophthalmol (2016).

[11] T. Itokawa, Y. Okajima, T. Suzuki, K. Kakisu, H. Iwashita, Y. Murakami, et al., Association between ocular surface temperature and tear film stability in soft contact lens wearers, Invest Ophthalmol Vis Sci 59 (2018) 771-775.

[12] R. Mapstone, Determinants of corneal temperature, Br J Ophthalmol 52 (1968) $729-741$.

[13] A.J. Bron, V.E. Evans, J.A. Smith, Grading of corneal and conjunctival staining in the context of other dry eye tests, Cornea 22 (2003) 640-650.

[14] J.J. Nichols, G.L. Mitchell, K.K. Nichols, R. Chalmers, C. Begley, et al., The performance of the contact lens dry eye questionnaire as a screening survey for contact lens-related dry eye, Cornea 21 (2002) 469-475.

[15] R.L. Chalmers, C.G. Begley, K. Moody, S.B. Hickson-Curran, Contact lens dry eye questionnaire-8 (CLDEQ-8) and opinion of contact lens performance, Optom Vis Sci 89 (2012) 1435-1442.

[16] M. Guillon, C. Maissa, Contact lens wear affects tear film evaporation, Eye Contact Lens 34 (2008) 326-330.

[17] J.J. Nichols, L.T. Sinnott, Tear film, contact lens, and patient factors associated with corneal staining, Invest Ophthalmol Vis Sci 52 (2011) 1127-1137.

[18] B. Long, J. McNally, The clinical performance of a silicone hydrogel lens for daily wear in an Asian population, Eye Contact Lens 32 (2006) 65-71.

[19] R.L. Chalmers, C. Hunt, S. Hickson-Curran, G. Young, Struggle with hydrogel Cl wear increases with age in young adults, Cont Lens Anterior Eye 32 (2009) 113-119.

[20] Q. Chen, J. Wang, M. Shen, L. Cui, C. Cai, M. Li, et al., Tear menisci and ocular discomfort during daily contact lens wear in symptomatic wearers, Invest Ophthalmol Vis Sci 52 (2011) 2175-2180.

[21] J.S. Wolffsohn, S. Mroczkowska, O.A. Hunt, P. Bilkhu, T. Drew, A. Sheppard, et al., Crossover evaluation of silicone hydrogel daily disposable contact lenses, Optom Vis Sci 92 (2015) 1063-1068.

[22] Y. Nagahara, S. Koh, N. Maeda, K. Nishida, H. Watanabe, Prominent decrease of tear Meniscus height with contact lens wear and efficacy of eye drop instillation,
Eye Contact Lens 41 (2015) 318-322.

[23] Q. Le, C. Jiang, A.C. Jiang, J. Xu, The analysis of tear meniscus in soft contact lens wearers by spectral optical coherence tomography, Cornea 28 (2009) 851-855.

[24] J. Wang, I. Cox, W.T. Reindel, Upper and lower tear menisci on contact lenses, Invest Ophthalmol Vis Sci 50 (2009) 1106-1111.

[25] M. Mousavi, D.A. Jesus, I.K. Garaszczuk, D.H. Szczesna-Iskander, D.R. Iskander, The utility of measuring tear film break-up time for prescribing contact lenses, Cont Lens Anterior Eye 41 (2018) 105-109.

[26] J. Varikooty, M.M. Schulze, K. Dumbleton, N. Keir, C.A. Woods, D. Fonn, et al., Clinical performance of three silicone hydrogel daily disposable lenses, Optom Vis Sci 92 (2015) 301-311.

[27] J. Wolffsohn, L. Hall, S. Mroczkowska, O.A. Hunt, P. Bilkhu, T. Drew, et al., The influence of end of day silicone hydrogel daily disposable contact lens fit on ocular comfort, physiology and lens wettability, Cont Lens Anterior Eye 38 (2015) 339-344.

[28] C. Purslow, J.S. Wolffsohn, J. Santodomingo-Rubido, The effect of contact lens wear on dynamic ocular surface temperature, Cont Lens Anterior Eye 28 (2005) 29-36.

[29] K.A. Dumbleton, C.A. Woods, L.W. Jones, D. Fonn, Comfort and adaptation to silicone hydrogel lenses for daily wear, Eye Contact Lens 34 (2008) 215-223.

[30] L.C. Thai, A. Tomlinson, M.G. Doane, Effect of contact lens materials on tear physiology, Optom Vis Sci 81 (2004) 194-204.

[31] E.H. Ooi, E.Y. Ng, C. Purslow, R. Acharya, Variations in the corneal surface temperature with contact lens wear, Proc Inst Mech Eng H 221 (2007) 337-349.

[32] E.B. Papas, C.M. Vajdic, R. Austen, B.A. Holden, High-oxygen-transmissibility soft contact lenses do not induce limbal hyperaemia, Curr Eye Res 16 (1997) 942-948.

[33] D. Fonn, K.E. MacDonald, D. Richter, N. Pritchard, The ocular response to extended wear of a high dk silicone hydrogel contact lens, Clin Exp Optom 85 (2002) $176-182$.

[34] D. Fonn, R. Peterson, C. Woods, Corneal staining as a response to contact lens wear, Eye Contact Lens 36 (2010) 318-321.

[35] S.W. Cheung, P. Cho, B. Chan, C. Choy, V. Ng, et al., A comparative study of biweekly disposable contact lenses: silicone hydrogel versus hydrogel, Clin Exp Optom 90 (2007) 124-131.

[36] V. Martín-Montañez, A. López-Miguel, C. Arroyo, M.E. Mateo, J.M. GonzálezMéijome, M. Calonge, et al., Influence of environmental factors in the in vitro dehydration of hydrogel and silicone hydrogel contact lenses, J Biomed Mater Res B Appl Biomater 102 (2014) 764-771.

[37] B.K. Nayak, Understanding the relevance of sample size calculation, Indian J Ophthalmol 58 (2010) 469-470. 